\title{
A Conceptual Framework to determine Medical Equipment Maintenance in Hospital Using RCM Method
}

\author{
Siti Hajar Salim ${ }^{1, *}$, Saiful Amri Mazlan ${ }^{2}$, Siti Aisyah Salim ${ }^{3}$ \\ ${ }^{1}$ Department of Management Technology, MJT, Kuala Lumpur, Malaysia. \\ ${ }^{2}$ Department of Mechanical Precision Engineering, UTM, Kuala Lumpur, Malaysia. \\ ${ }^{3}$ Faculty of Technology Management and Business, UTHM, Batu Pahat, Johor, Malaysia.
}

\begin{abstract}
Maintenance services are performed to ensure the equipment is operating according to manufacturer standard and maintained at a desired level of quality. The implementation of maintenance services may prevent unexpected breakdown and disruption of operations in a hospital. However, there were numbers of cases reported on the frequent breakdown of medical equipment even though the maintenance program is in place and practised. Therefore, the reliability of the maintenance services was questioned especially when the breakdowns occurred after the maintenance services being performed. This paper proposes a conceptual framework to determine the accurate maintenance program for medical equipment in the hospital using Reliability Centred Maintenance (RCM) method. The recommendation from the RCM will assist to identify the root cause of the breakdown and minimize the number of breakdown for medical equipment in the hospital. RCM process is known as one of the most effective maintenance approaches to reduce the number of maintenance activities and hence minimizing the cost of maintenance incurred.
\end{abstract}

\section{Introduction}

Medical equipment plays a vital role in the diagnosis, monitoring and treatment patient in a hospital facility. As important as it has been, the hospital needs to maintain rigorous standards and requirements of medical equipment to ensure the safety of patients and the staff. Absence or failure of medical equipment could significantly pull down the medical industry and become detrimental to the lives of billions of people worldwide. Consequently, to ensure medical equipment is well maintained in good and safe condition, maintenance program needs to be performed. A maintenance program of medical equipment is crucially important not only to ensure that the equipment is maintained at its best condition but also to avoid any failures or breakdown occurred during its operations. Furthermore, maintenance is considered as "profit contributor" and "partner" for world-class competitiveness [1].

In many cases, maintenance program in the hospital is not optimized but rather simply being recommended by manufacturer or expert with operational experiences [2]. Therefore, the hospital might not select the right maintenance program to their organization and they may pay the unnecessary cost in maintaining the equipment. The problem widens up when frequent equipment breakdown occurred despite all maintenance program being implemented. These breakdowns were sometimes rather being corrected than to find the original cause of the problems. Problemsolving is only achievable when the cause of the breakdowns being identified [3]. Breakdown not only may happen due to the system itself but skills and behavioural factor can affect the performance of the equipment [4]. Equipment downtime due to breakdown will affect the productive capability of equipment by reducing output, increasing operating costs and interfering with customer service. Therefore, to solve these issues, we are proposing a conceptual framework to identify the right maintenance program to be implemented in the hospital by identifying the cause of the breakdown by using Reliability Centred Maintenance (RCM).

RCM is one of the most effective maintenance approaches capable of reducing maintenance activities and their related costs without affecting the overall performance of the equipment, quality, safety and environmental integrity [5]. RCM has been widely and successfully used in vast industries especially in industries with the complex system [6] such as military, navy, nuclear power plant, electrical power generation and several other sectors [7]. RCM is practical to be used in this study as it focuses on the functional failures of systems and components [8]. Recurrent breakdowns can only be resolved if thorough investigation employed on the system by going in-depth into components of the equipment [9]. Therefore, RCM can be used to find the optimal maintenance and operational strategies based on the probability and consequences of the analysis failure modes [10]. RCM is used as a new strategic framework for ensuring that any asset continues to perform, as its users want it to perform [11].

Corresponding author: hajarsalimalbajrai@gmail.com 
Consequently, introducing RCM in the healthcare industry will enhance the quality of maintenance implementation and thus increase the reliability of medical equipment in service [12]. RCM is consists of principles that are to ensure the effectiveness of the maintenance approach. These principles are function oriented, systems focused, acknowledge design limitations, driven by safety and economics, and define failure as any unsatisfactory condition and uses the logic tree to screen maintenance tasks [13]. This framework is highly recommendable for high-end equipment such as imaging equipment (i.e. Magnetic Resonance Imaging, Computed Tomography etc) and radiotherapy equipment (i.e. Positron Emission Tomography, Linear Accelerator) as the downtime will greatly impact the medical process in the hospital.

\section{Research Method}

The objective of this conceptual framework is to determine the right maintenance services for medical equipment using RCM. The causes of breakdown need to be first identified in order for a solution to be suggested. RCM process is then to be implemented where the last step of RCM is to recommend a new process wherein this context is the new maintenance services program, by comparing with the current practice of maintenance services.

\subsection{Breakdown Factors}

Breakdown of medical equipment may occur due to factors namely; maintenance services type factor, environmental factor, human factor and the condition of equipment factor. These factors were identified by interviewing five biomedical engineers with more than five years experiences in the hospital. Only five engineers were selected for this study as most of them gave common answers. Five common factors will be discussed as follow.

Table 1. Factors of medical equipment breakdown

\begin{tabular}{|c|c|c|}
\hline No & Factors of breakdown & Descriptions \\
\hline 1. & $\begin{array}{l}\text { Maintenance services } \\
\text { type factors }\end{array}$ & 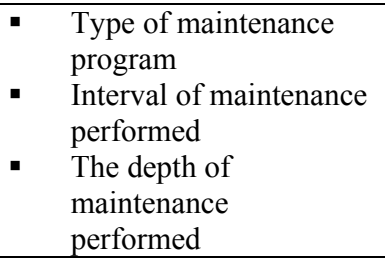 \\
\hline 2. & Environmental factors & $\begin{array}{l}\text { - } \quad \text { Stability of power supply } \\
\text { - Temperature and } \\
\text { humidity }\end{array}$ \\
\hline 3. & Human factors & $\begin{array}{l}\text { - } \quad \text { Lack of skills } \\
\text { - } \quad \text { Misuse of equipment }\end{array}$ \\
\hline
\end{tabular}

Based on these factors, we were only focusing on maintenance services type and elaborate in details in proposing the conceptual framework. The environmental factor is much depending on external causes while human factors are something that technically cannot be controlled and therefore it is ruled out from this proposal.

Type of maintenance may contribute the stability and the reliability of medical equipment system as the wrong implementation of maintenance services program may result in system breakdown. Zero breakdown of medical equipment is almost impossible to achieve an external factor or sudden changes in the system might impact the stability of the equipment thus lead to the failures. However, breakdown due to parts problems is attainable to be minimized if a correct maintenance program being applied [14]. From the interview session, we found one similarity area of concerns of the engineers on the current maintenance program in their hospital which is the reliability of the maintenance itself. The number of breakdown for high-end equipment is relatively high per year though well-planned maintenance services are in place. This is relatively in line with the purpose of our study to propose a conceptual framework to propose a right maintenance services program and to identify the root of recurrent equipment breakdown, especially for high-end medical equipment.

\subsection{Reliability-Centred Maintenance Conceptual Framework Proposal.}

The RCM analysis processes focus on the functions of equipment, the consequences of failure and measures to prevent or cope with functional failure. The process of RCM involves the step as follows [15]:

Table 2. Reliability-Centred Maintenance Steps

\begin{tabular}{|c|c|}
\hline $\begin{array}{l}\text { Reliability-Centred } \\
\text { Maintenance Steps }\end{array}$ & Description \\
\hline $\begin{array}{l}\text { Step 1: Data } \\
\text { preparation }\end{array}$ & $\begin{array}{ll}\text { - } & \text { System selection } \\
\text { - } & \text { Data collection } \\
\text { - } & \text { System boundaries }\end{array}$ \\
\hline Step 2: Analysis. & $\begin{array}{ll}\text { - } & \text { Equipment identification } \\
\text { - } & \text { Identification of system } \\
\text { functions } \\
\text { - } & \text { Failure mode effect analysis } \\
\text { - } & \begin{array}{l}\text { Determination of component } \\
\text { criticality }\end{array} \\
\text { - } & \text { Logic tree diagram }\end{array}$ \\
\hline Step 3: Task selection. & $\begin{array}{l}\text { - } \quad \text { Continuous predictive } \\
\text { maintenance } \\
\text { - } \text { Scheduled predictive } \\
\text { maintenance } \\
\text { - } \quad \text { Overhaul } \\
\text { - } \quad \text { Replacement } \\
\text { - Inspection and functional } \\
\text { testing and Run-to-Failure }\end{array}$ \\
\hline $\begin{array}{l}\text { Step 4: Task } \\
\text { comparison and review }\end{array}$ & $\begin{array}{l}\text { Recommendation of new } \\
\text { task/ process selection } \\
\text { comparing with the current } \\
\text { practice } \\
\text { - The output of analysis to }\end{array}$ \\
\hline
\end{tabular}




\begin{tabular}{|l|l|}
\hline & $\begin{array}{l}\text { change maintenance } \\
\text { program. }\end{array}$ \\
\hline
\end{tabular}

\subsubsection{Step 1: Data Preparation}

The data to be collected based on system selection; type of equipment selected. The selection is based on the criticality in availability and functionality. For example, Computed Tomography scanner (CT scanner) is selected as the absence of equipment will disturb the process in the hospital, and the equipment is critically important when urgent scanning needed for critical cases. In the selection equipment for the data collection, system boundaries need to be defined for further analysis. System boundaries will define the necessary information to be added and what are to be omitted from the data analysis.

\subsubsection{Step 2: Analysis}

The analysis can be commenced once the systems have been selected. The data must comprehensive collected from the point where the equipment being installed in the hospital until the current time where the analysis starts.

\subsubsection{Equipment identification and its functions}

Equipment that has been selected for the analysis must be profoundly understood especially their structure of equipment, the applications and functions. This is an initial step where we need to know the principle of the system and their function before any action or method can be implemented.

\subsubsection{Failure mode effect analysis (FMEA)}

Failure mode and effect analysis (FMEA) is a tool that examines the potential product or process failures, evaluates risk priorities, and helps determine remedial actions to avoid identified problems [16]. FMEA is one of the risk analysis techniques that have been widely used to identify potential failures [17]. It is a systematic process that highly recommended by international standards that able to identify possible failure causes so that the causes can be eliminated and to locate the failure's impacts so the impacts can be reduced [18]. An application of a FMEA follows a series of successive step: analysis of the process, product or system in every single part, listing of identified potential failures, evaluation of their frequency, severity and detection technique, global evaluation of the problem and identification of the corrective actions and control plans that could eliminate or reduce the chance of the potential failures [19] [20]. An FMEA uses risk priority number (RPN) to assess risk in three categories (Fig. 1)

i) Occurrence $(\mathrm{O})$ - the assessment of how frequently the specific failure cause is projected to occur,

ii) Severity (S)- the assessment of the seriousness of the effect of the potential failure to the system and iii) Detection (D) - the assessment of the probability that the operating parameters monitoring system will detect a cause/mode of failure before the component/system is damaged and stopped.

$$
R P N=O \times S \times D
$$

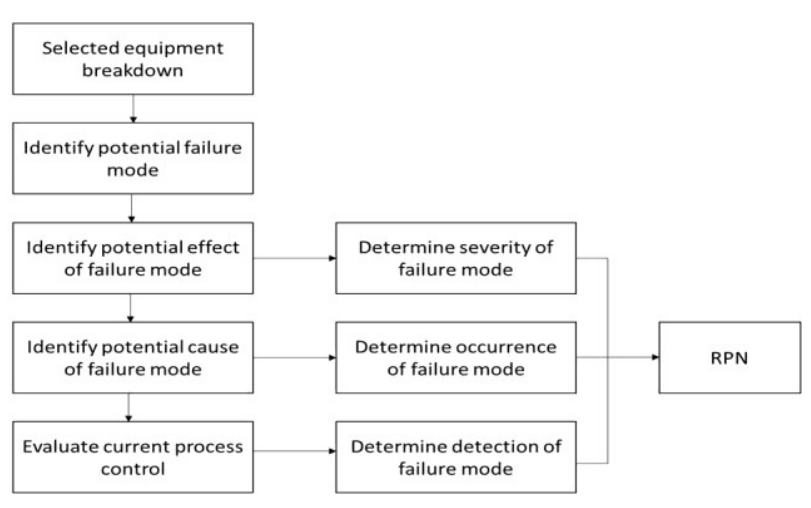

Fig. 1. The process of the FMEA method [21]

\subsubsection{Determination of Component Criticality}

A reliability centred maintenance approach requires identification of the critical components of the system. A component is defined as critical if its failure effects are massive to the equipment. For example, the x-ray tube is very important to $\mathrm{CT}$ scanner whereby faulty of the part will the effect the whole system from being functioning thus equipment cannot be operated. Medical equipment is usually composed of a variety of electronic parts which categorized into parts that greatly impacting the equipment and parts that are less impacting the equipment. The hospital needs to identify parts that are critically important based on a recommendation by the manufacturer or authorized vendor/ distributors. This will help the monitoring process to be focused and narrowed down on the critical parts of equipment

\subsubsection{Logic tree diagram}

RCM utilizes a decision logic tree to identify the maintenance requirements of equipment according to the safety and operational consequences of each failure and the degradation mechanism responsible for the failures [22].

The Logic Tree Analysis (LTA) process is used to determine the most applicable, cost-effective preventive maintenance tasks for a component in equipment [23]. These recommended tasks are typically a function of component importance, design, utilization and service environment. 


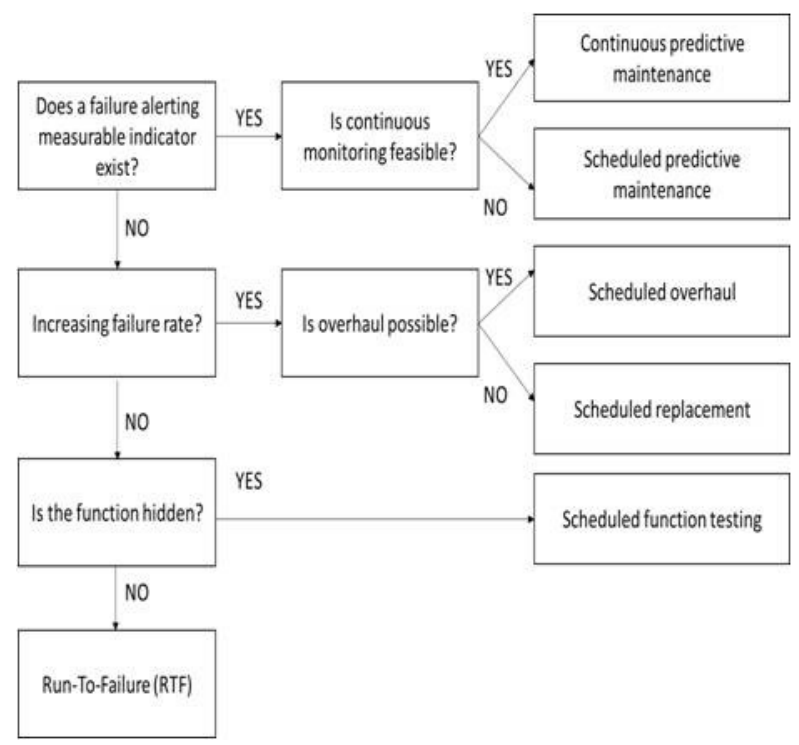

Fig. 2. Example of RCM Logic Tree Analysis [24]

\subsubsection{Step 3: Task Selection}

Task selection needs to be determined based on Logic Tree Analysis (Figure 2). The tasks selected are influenced by the failure causes and appropriate to be implemented for the respective equipment. Medical equipment system can be very simple or complicated therefore the maintenance program for each of the system might vary. Task selection is much depending on the type of equipment and the availability of support given by the manufacturer. The cost of maintenances needs to be minimized without compromising with the reliability and availability of the medical equipment. The task selection must be made thoroughly as wrong selection of task might as increase the maintenance cost and reducing the reliability

\subsubsection{Step 4: Task comparison and review}

The effectiveness of a task can only be accessed by comparing the results of before and after the implementation. The number of breakdown with the current maintenance program implemented in the hospitals need to be compared with the number of breakdowns after the new proposed maintenance program being implemented. The result of the comparison can be reviewed and can determine whether the hospital made the right task selection or contrary. Further improvement may be addressed and introduced based on the review of the results.

\section{Discussion}

In general, medical equipment breakdown will not only impact the operational and financial of the hospital but also affecting the safety of the patient especially to a patient who needs to seek emergency treatment and attention. Unavailability of equipment especially those that fall under high-risk equipment may endanger the life of the patient if immediate actions are not being taken.
Equipment breakdown due to parts or system failure is a common issue in the hospital through most the hospitals are practicing well-developed maintenance program to ensure the stability and availability of equipment. This maintenance program is sometimes cost the hospital more the purchasing price itself especially when involves in major breakdown problems. Therefore, the hospital sector need to ensure that the money that they spent on the maintenance program in their hospital is worth the spending. Hospitals need to avoid paying the unnecessary cost in maintaining medical equipment in their organization and to ensure the maintenance program implemented in their organization in compliance with standard and requirements without compromising with the safety of the patient. This conceptual framework not only will help the hospital to determine the right maintenance services to be practiced but also will assist the hospital to identify the root cause of the medical equipment breakdown.

\section{Limitations}

RCM is widely accepted methodology that has been available in the industry for over 30 years and has proved to offer an efficient maintenance program [25] however many organizations failed to achieve their goal due to lack of data and gave up halfway as the process is long and lengthy. The effectiveness of RCM can be obtained in a long run process where effort and time need to be spent lavishly to achieve the target. Some organizations lack expert especially in monitoring and analysing the data. For medical equipment industry specifically, some of the data need to be obtained from the Original Equipment Manufacturer (OEM) but not all have the data and even those who have the data might not want to share the data to the hospital.

\section{Conclusions}

RCM is a reliable decision-making tool. The principle of RCM is doing the right work at the right time based on equipment condition. The outcome of an RCM analysis may result in changes to existing maintenance tasks, where it will enhance the safety and reliability of the equipment and the optimization and maintenance activity. If RCM performed effectively, it will result in the elimination of unnecessary maintenance tasks and the introduction of measures to address omissions and deficiencies in maintenance programmes. Therefore, not only the quality of the services provided by the hospital is at its highest value, but their medical equipment as well is able to be maintained at minimum cost without compromising with the safety requirements.

\section{References}

1. Waeyenberg et al., A framework for maintenance concept development. International Journal of Production Economics. Vol.77, No.1, pp. 299-313 (2002) 
2. N. Bloom, Reliability centred maintenance: implementation made simple. NY: McGraw-Hill Companies, (2006)

3. M. A. Rausand, System Reliability Analysis: Models, Statistical Methods And Applications. 2nd ed. Hoboken N.J Wiley- Interscience, (2003)

4. V. Narayan. Business performance and maintenance: how are safety, reliability, productivity and maintenance related. Journal of Quality Maintenance Engineering, Vol 18, Issue 1, pp. 183-195, (2012)

5. V.S. Desphande et al., Application of RCM to a medium scale industry. Journal of Reliability Engineering and System Safety. Vol 77, Issue 2, pp. 31-43, (2002)

6. X. Jia et. al. A prototype cost model of functional check decisions in reliability-centred maintenance, Journal of the Operational Research Society, Vol 53, Issue 12, pp. 1380-1384, (2012).

7. F. Backlund et al., RCM introduction: process and requirements management aspects, Journal of Quality in Maintenance Engineering, Vol 9, Issue 3, pp. 250-264, (2003)

8. A. Saniuk, et al., Environmental favourable foundries through maintenance activities. Journal of Metalurgija, Vol 54, Issue 4, pp. 725-728, (2015)

9. M. Rausand, Reliability-Centered Maintenance. Reliability Engineering and System Safety, Vol 60, Issues 2, pp. 121-132, (1998)

10. S.I. Mostafa, Implementation of proactive maintenance in the Egyptian glass company, Journal of Quality in Maintenance Engineering, Vol 10, Issue 2, pp.107-122, (2014)

11. V.S. Desphande et al., Application of RCM to a medium scale industry. Journal of Reliability Engineering and System Safety. Vol 77, Issue 2, pp. 31-43, (2002)

12. G. H. Ronda, Patient safety and quality. An Evidence-Based Handbook for Nurses. Rockville (MD): Agency for Healthcare Research and Quality (US), (2008)

13. A. Kelly, Maintenance strategy, business centred maintenance, Oxford: Reed Educational and Professional Publishing, (1998)

14. A. H. Tsang, Condition-based maintenance: tools and decision making. Journal of Quality in Maintenance Engineering, Vol 1, Issue 3, pp. 3-17. ISSN 1355-2511, (1995)

15. International Atomic Energy Agency (IAEA) Application of centred maintenance to optimize operation and maintenance in nuclear plants. Nuclear Power Engineering Section International Atomic Energy Agency, Vienna Austria, 2007.

16. I. H. Afefy, Reliability-centered maintenance methodology and application: a case study. Journal of Scientific Research, Vol 1, Issue 1, pp. 863-873, (2010)

17. Z. XiuXiu et. al. The application of FMEA method in the risk management of medical device during the lifecycle. IEEE, 2010.

18. Guidelines for Failure Mode and Effects Analysis for Automotive, Aerospace and General
Manufacturing Industries, Dyadem Press, Ontario Canada, 2003.

19. G. Ireson et al., Handbook of Reliability Engineering and Management - 2nd ed., McGrawHill Professional, New York, (1995).

20. A. Scipioni et. al. FMEA methodology design, implementation and integration with the HACCP system in a food company. Food Control, Vol 13, Issue 1, pp. 495- 501, (2002)

21. Małgorzata et al., SWOT analysis for Planned Maintenance strategy. Journal of International Federation of Automatic Control, 49(12),pp. 674679, (2016)

22. J. Vatn, Maintenance in the rail industry. Preventive maintenance model for complex systems. In: Kobbacy KAH, Murthy DNP editors. Complex System Maintenance Handbook. London SpringerVerlag Limited, Chapter 12: p. 509-532, (2008)

23. V.S. Desphande et al., Application of RCM to a medium scale industry. Journal of Reliability Engineering and System Safety. 77(2): p. 31-43, (2002) 